BMJ Open

Diabetes

Research

\& Care

\section{Insulin resistance, beta-cell function, adipokine profiles and cardiometabolic risk factors among Chinese youth with isolated impaired fasting glucose versus impaired glucose tolerance: the BCAMS study}

Yu Li, ${ }^{1}$ Dan Feng, ${ }^{2}$ Issy C Esangbedo, ${ }^{3}$ Yanglu Zhao, ${ }^{4}$ Lanwen Han, ${ }^{2}$ Yingna Zhu, ${ }^{2}$ Junling Fu, ${ }^{1} \mathrm{Ge} \mathrm{Li},{ }^{1}$ Dongmei Wang, ${ }^{1}$ Yonghui Wang, ${ }^{2}$ Ming Li (D) , ${ }^{1}$ Shan Gao, ${ }^{2}$ Steven M Willi (D) ${ }^{5}$

To cite: Li Y, Feng D, Esangbedo IC, et al. Insulin resistance, beta-cell function, adipokine profiles and cardiometabolic risk factors among Chinese youth with isolated impaired fasting glucose versus impaired glucose tolerance: the BCAMS study. BMJ Open Diab Res Care 2020;8:e000724. doi:10.1136/ bmjdrc-2019-000724

- Additional material is published online only. To view, please visit the journal online (http://dx.doi.org/10.1136/ bmjdrc-2019-000724).

$\mathrm{ML}$ and $\mathrm{SG}$ contributed equally.

YL and DF are joint first authors.

Received 30 June 2019 Revised 8 January 2020 Accepted 19 January 2020

Check for updates

(c) Author(s) (or their employer(s)) 2020. Re-use permitted under CC BY-NC. No commercial re-use. See rights and permissions. Published by BMJ.

For numbered affiliations see end of article.

Correspondence to

Professor Ming Li;

liming@pumch.cn

Professor Shan Gao; gaoshanmw@163.com

\section{ABSTRACT}

Objective Impaired fasting glucose (IFG) and impaired glucose tolerance (IGT) may convey disparate risks of metabolic consequences. Fasting plasma glucose (FPG), while an expedient screening procedure, may not adequately assess metabolic risk, particularly among youths. In order to inform a strategy for screening Chinese youth for pre-diabetes, we examined the relative value of IFG versus IGT to define metabolic risk by assessing their association with insulin resistance, beta-cell dysfunction, adverse adipokine profiles and other cardiometabolic risk factors.

Research design and methods We recruited 542 subjects (age 14-28 years) from the Beijing Child and Adolescent Metabolic Syndrome study for an in-depth assessment of cardiometabolic risk factors, including a 2-hour oral glucose tolerance test, liver ultrasound and serum levels of four adipokines.

Results FPG failed to identify nearly all (32/33) youths with IGT, whereas 2-hour plasma glucose (2h PG) missed $80.8 \%(21 / 26)$ of subjects with IFG. Impaired beta-cell function was evident from decreased oral disposition indices in those with isolated impaired fasting glucose (ilFG) or isolated impaired glucose tolerance (ilGT) versus normal glucose tolerance (NGT) (all $p<0.001$ ), whereas reduced insulin sensitivity (Matsuda) index was most pronounced in the ilGT group $(p<0.01)$. Moreover, alterations in adipokine levels (fibroblast growth factor 21, adiponectin and leptin/adiponectin ratio) were associated with ilGT $(p<0.05)$ but not ilFG. Youths with ilGT had a 2fold to 32-fold increased incidence of hypertriglyceridemia, hypertension and metabolic syndrome (MetS) compared with those with NGT. In addition, subgroup analyses of participants with normal FPG revealed that the odds of having IGT increased 3-fold to 18-fold among those with elevated TGs, hypertension, moderate-to-severe nonalcoholic fatty liver disease or MetS.

Conclusions Chinese youth with ilGT exhibit a higher cardiometabolic risk profile than those with ilFG. Thus, $2 \mathrm{~h} P \mathrm{PG}$ is preferred over FPG to identify the pre-diabetes

\section{Significance of this study}

What is already known about this subject?

- Impaired fasting glucose and impaired glucose tolerance (IGT) may reflect different metabolic subphenotypes of pre-diabetes, which may carry disparate risks of metabolic consequences.

- Fasting plasma glucose (FPG) is recommended for screening for pre-diabetes due to cost and convenience compared with 2-hour oral glucose tolerance test and hemoglobin A1C.

What are the new findings?

- The IGT phenotype is associated with a more adverse cardiometabolic risk profile and may be a stronger predictor of cardiovascular disease than the impaired fasting glucose phenotype in youth.

- Alterations in adipokine concentrations are already detectable in IGT states and may reflect adipose tissue dysfunction as an early pathogenic event in the development of type 2 diabetes.

- FPG is inadequate for the identification of IGT and its accompanied elevated cardiovascular risk in youth.

How might these results change the focus of research or clinical practice?

$>$ Our findings suggest that a 2-hour plasma glucose rather than FPG test is necessary to identify the prediabetes phenotype among youths at risk of metabolic syndrome.

phenotype at greatest risk of subsequent development of cardiovascular disease.

Trial registration number NCT03421444.

\section{INTRODUCTION}

The increasing prevalence of type 2 diabetes (T2D) among young individuals, both globally 
and in China, presents a major public health challenge. In 2017, the International Diabetes Federation (IDF) estimated that 425 million people between the ages of 20 and 79 years worldwide were affected by T2D, with the number projected to increase to 629 million by $2045 .{ }^{1}$ According to a recent national survey in China, nearly 1 in 10 adults had T2D and the prevalence of pre-diabetes was as high as $50 \% .^{2}$ As T2D is a progressive disease with insidious onset, often silent until presenting with organ damage after many years of hyperglycemia, screening programs for early manifestations of the disease, like prediabetes, are needed. ${ }^{3}$

Pre-diabetes is defined as an intermediate metabolic state between normal glucose tolerance (NGT) and overt T2D, which encompasses individuals with impaired fasting glucose (IFG) as well as impaired glucose tolerance (IGT) ${ }^{4}$ Studies in adults have suggested the existence of metabolic subphenotypes among pre-diabetic individuals, with IFG being characterized by a combination of hepatic insulin resistance (IR) and defective earlyphase insulin secretion, while IGT primarily consists of peripheral IR combined with impaired first-phase and second-phase insulin secretion. ${ }^{5-9}$ Not only is pre-diabetes an intermediate state of dysglycemia, but also it exists on a continuum of cardiometabolic risk between metabolic syndrome (MetS) and T2D. ${ }^{10}{ }^{11}$ However, the overlapping pathogeneses of dysglycemia and cardiometabolic dysfunction are not fully understood. While this overlap can be partially attributed to an intricate relationship between metabolism and inflammation, ${ }^{12}{ }^{13}$ a number of adipokines, such as leptin, adiponectin, fibroblast growth factor 21 (FGF21), and retinol binding protein 4 (RBP4) play an important role not only in the atherosclerotic process but also in the development of diabetes. ${ }^{14} 15$ Nonetheless, the preponderance of evidence addressing the pathophysiological mechanisms responsible for a relationship between cardiometabolic risk and prediabetes are derived from adult-based studies, with little understanding of the clinical manifestations in youth, especially from China. While fasting plasma glucose (FPG), 2-hour oral glucose tolerance test (OGTT) and hemoglobin A1c (HbAlc) are all endorsed by the American Diabetes Association (ADA) to test for pre-diabetes and diabetes, FPG is preferred as a screening test due to cost and convenience, especially in youth, ${ }^{16}{ }^{17}$ despite the fact that its efficiency is still under debate.

Given that little is known about the evolution of these conditions in young populations and the likely existence of metabolic subphenotypes within pre-diabetes, with differing abilities to be detected and to portend cardiovascular disease, the aims of the present study were to (1) examine the efficacy of IFG as a screening strategy for identifying IGT in adolescents and young adults, (2) investigate the characteristics of insulin sensitivity and pancreatic beta-cell function in youth with isolated IFG versus IGT, (3) compare adipokine and cardiometabolic risk profiles between those with isolated IFG and IGT, and (4) compile these data to inform a strategy for screening at-risk youth for pre-diabetes based on data from the Beijing Child and Adolescent Metabolic Syndrome (BCAMS) study. ${ }^{18-20}$

\section{METHODS}

Participants

The BCAMS is a longitudinal cohort study of cardiovascular risk factors beginning in childhood. The study protocol has been reported in detail elsewhere ${ }^{18-21}$ and is registered online (www.clinicaltrails.gov). Briefly, in 2004, a population-based survey was conducted in the Beijing area with a representative sample of children and adolescents ( $\mathrm{n}=19593,50 \%$ boys). Approximately 4500 were identified as being at elevated risk of MetS due to the presence of at least one of the following risk factors: overweight defined by a body mass index (BMI) at $\geq 85$ th percentile; blood pressures (BPs) at $>90$ th percentile, total cholesterol of $\geq 5.2 \mathrm{mmol} / \mathrm{L}$, triglyceride (TG) of $\geq 1.7 \mathrm{mmol} / \mathrm{L}$ or $\mathrm{FPG}$ of $\geq 5.6 \mathrm{mmol} / \mathrm{L}$ based on capillary blood sampling. Beginning in 2012, these at-risk individuals were recruited consecutively through various modalities (phone, text and email) for a medical examination. ${ }^{19}$ Among this follow-up cohort of 559 individuals $(14-28$ years, mean $=20.2$ years $), 17$ did not consent to take OGTT, leaving 542 available for the current analysis.

\section{Clinical measurements}

Anthropometric measurements, including height, weight, waist circumference (WC), and fat mass percentage (FAT\%), were measured following standardized procedures ${ }^{19} 20$ after removing bulky clothing and shoes. Height was measured to the nearest millimeter using a portable stadiometer. WC was measured midway between the lowest rib and the top of the iliac crest. Weight and FAT\% were measured to the nearest $0.1 \mathrm{~kg}$ using a TANITA Body Composition Analyzer (ModelTBF-300A). Measurements of systolic blood pressure (SBP) and diastolic blood pressure (DBP) were recorded in the sitting position from the right arm with a mercury sphygmomanometer and rounded to the nearest $2 \mathrm{~mm} \mathrm{Hg}$. Three readings were taken $10 \mathrm{~min}$ apart, and the mean of the lowest two readings was taken as the BP. BMI was calculated as weight divided by height squared. Non-alcoholic fatty liver disease (NAFLD) was diagnosed by ultrasonography according to the 2010 Prevention and Treatment Guidelines for NAFLD published by the Chinese Liver Disease Association ${ }^{22}$ and was graded as mild, moderate or severe according to the ultrasonographic images by referring to established criteria ${ }^{2324}$ : mild, characterized by mildly increased liver echogenicity and clear depiction of hepatic and portal vein walls; moderate, with increased liver echogenicity obscuring the hepatic and portal vein walls; and severe, with increased liver echogenicity and significant posterior shadowing that impairs evaluation of the deep liver parenchyma and diaphragm. 


\section{Laboratory measurements}

After a 12-hour overnight fast, participants performed a standard OGTT (with $1.75 \mathrm{~g} / \mathrm{kg}$, maximum $75 \mathrm{~g}$ glucose). Blood samples were obtained for the measurement of glucose and insulin at $0,0.5$, and 2 hours after oral glucose administration. The samples were centrifuged, aliquoted and immediately frozen for future analysis of hormones. HbA1c was analyzed using the TOSOH G7 automated analysis system with high-performance liquid chromatography. Serum lipids and glucose were assayed using the Hitachi 7060 C automated biochemistry analyzer. Lowdensity lipoprotein cholesterol (LDL-C) and high-density lipoprotein cholesterol (HDL-C) levels were measured directly. High-sensitivity $\mathrm{C}$ reactive protein was measured by immunoturbidimetric assay. Serum aspartate transaminase and alanine amino transferase were assayed using an automated biochemistry analysis system. Insulin, leptin and adiponectin concentrations were measured by monoclonal antibody-based sandwich ELISA developed in the Key Laboratory of Endocrinology, Peking Union Medical College Hospital. ${ }^{25-27}$ The intra-assay coefficients of variation (CVs) for insulin, leptin and adiponectin were $<4.1 \%,<7.4 \%$ and $<5.4 \%$, respectively. The interassay CVs were $<7.0 \%,<9.3 \%$ and $<8.5 \%$, respectively. FGF21 and RBP4 were measured using a commercial ELISA Kit (R\&D Systems, Minnesota, USA) with interassay CVs of $<7.4 \%$ and $<10 \%$, respectively. ${ }^{28}{ }^{29}$ All samples were analyzed in duplicate.

\section{Calculations and definitions}

Using blood glucose and serum insulin levels from the OGTT, we calculated the homeostasis model assessment of insulin resistance (HOMA-IR, mainly reflecting hepatic IR) as HOMA-IR=fasting insulin (FIns, mU/L) $\times$ FPG $(\mathrm{mmol} / \mathrm{L}) / 22.5,{ }^{30}$ and whole-body insulin sensitivity (Matsuda) index (ISI ${ }_{\text {Matsuda }}$, reflecting both hepatic and peripheral insulin sensitivity) during the OGTT calculated as $\mathrm{ISI}_{\text {Matsuda }}=10000 /(\mathrm{FPG} \times \mathrm{FIns}) \times(\mathrm{G} \times \mathrm{I}),{ }^{31}$ where $\mathrm{G}=$ mean serum glucose and $\mathrm{I}=$ mean serum insulin concentration. These indices have been demonstrated to strongly correlate with the euglycemic-hyperinsulinemic clamp and minimal-model frequently sampled intravenous glucose tolerance test-derived measures of insulin sensitivity in obese children and adolescents. ${ }^{32-34}$ Pancreatic beta-cell function was estimated using the following indices: Homeostasis model assessment of $\beta$-cell function $($ HOMA- $\beta)=20 \times$ FIns $/($ FPG -3.5$),{ }^{30}$ insulinogenic index $(\mathrm{IGI}=\Delta$ Ins30 $/ \triangle$ Gluc30 $),{ }^{35}$ and oral disposition index $($ DIo $=$ IGI $\times$ ISI $),{ }^{36}$ which reflects the capacity of pancreatic islets to compensate for reduced insulin sensitivity.

Glucose tolerance status was defined according to ADA criteria, ${ }^{37}$ with subjects classified as having pre-diabetes, including the following: IFG: $\mathrm{FPG} \geq 5.6-7.0 \mathrm{mmol} / \mathrm{L}$ and IGT: 2-hour plasma glucose (2h PG) $\geq 7.8-11.1 \mathrm{mmol} / \mathrm{L}$. T2D was diagnosed in patients with $\mathrm{FPG} \geq 7.0 \mathrm{mmol} / \mathrm{L}$, OGTT $2 \mathrm{~h} \mathrm{PG} \geq 11.1 \mathrm{mmol} / \mathrm{L}$ or $\mathrm{HbAlc} \geq 6.5 \%$. MetS was defined according to the harmonized criteria when at least three of the following five criteria were met $^{38}:(1)$ central obesity: $\mathrm{WC} \geq 90$ th in $10-16$ years, $\mathrm{WC} \geq 90 \mathrm{~cm}$ for boys and $\mathrm{WC} \geq 80 \mathrm{~cm}$ for girls in over 16year olds; (2) $\mathrm{FPG} \geq 5.6 \mathrm{mmol} / \mathrm{L}$ and $/$ or $2 \mathrm{~h} \mathrm{PG} \geq 7.8 \mathrm{mmol} / \mathrm{L}$ or a diagnosis of diabetes; (3) $\mathrm{SBP} \geq 130 \mathrm{~mm} \mathrm{Hg}$ or $\mathrm{DBP} \geq 85 \mathrm{~mm}$ $\mathrm{Hg}$ or presence of drug treatment for hypertension; (4) HDL-C< $<1.03 \mathrm{mmol} / \mathrm{L}$ in men or $<1.29 \mathrm{mmol} / \mathrm{L}$ in women; and (5) TG $\geq 1.70 \mathrm{mmol} / \mathrm{L}$.

\section{STATISTICAL ANALYSIS}

All statistical analyses were carried out using the Statistical Package for Social Sciences V.21.0 for Windows. Data are presented as mean $\pm \mathrm{SD}$ or geometric mean (with 95\% CI) for continuous parameters and percentage for categorical variables. All skewed distributions underwent natural logarithmic transformation prior to analysis. Comparisons between groups were accomplished using t-tests or analysis of variance with post hoc comparison tests for continuous variables, while categorical variables were explored using the $\chi^{2}$ test. Logistic regression analysis was used to calculate the OR and CI of variables while adjusting for possible confounders. The $\mathrm{k}$ statistic was calculated as a measure of agreement between FPG and 2-hour OGTT diagnoses for individuals with hyperglycemia. A p value of $<0.05$ (two-sided) was considered to be statistically significant.

\section{RESULTS}

\section{Agreement of classification between FPG and 2 h PG during} OGTT

We evaluated the agreement of classification between FPG and $2 \mathrm{~h}$ PG during OGTT (online supplementary table 1). Of the total 542 participants, $54(10 \%)$ had pre-diabetes, including $21(3.9 \%)$ with isolated impaired fasting glucose (iIFG), 32 (5.9\%) with isolated impaired glucose tolerance (iIGT) and one $(0.2 \%)$ with combined IFG/IGT, while 10 (1.9\%) met the criteria for T2D. There was poor agreement between the classification of prediabetes and diabetes defined by FPG and $2 \mathrm{~h}$ PG during OGTT (kappa value of 0.12 ). FPG failed to identify the majority $6 / 10(60 \%)$ of those with T2D and nearly all $32 / 33(97 \%)$ with IGT, whereas 2 h PG would miss 21/26 $(80.8 \%)$ of individuals with IFG.

\section{Characteristics of the participants stratified by NGT, ilFG and ilGT}

We next focused on participants with iIFG and iIGT. The clinical and biochemical features of the study participants grouped as NGT, iIFG and iIGT are shown in table 1 . The three groups were similar in gender and age distribution. Compared with the NGT group, the iIFG group had higher BMI $(p<0.05)$, WC $(p<0.05)$, FAT\% $(p<0.05)$, TG $(p<0.001)$, LDL-C $(p<0.05)$, DBP $(p<0.05)$ and HbA1c $(\mathrm{p}<0.001)$, while iIGT had higher FAT\% $(\mathrm{p}<0.05)$, SBP $(\mathrm{p}<0.05)$ and DBP $(\mathrm{p}<0.05)$ than NGT and lower TG $(\mathrm{p}<0.05)$ than the iIFG group. FIns was highest in the iIFG group, and 2-hour insulin levels reached their peak in the iIGT group. The prevalence rates of MetS 
Table 1 Characteristics of the study participants according to glucose homeostasis status

\begin{tabular}{|c|c|c|c|c|}
\hline & NGT & iIFG & IIGT & $P$ value \\
\hline Gender (male/female) & $252 / 226$ & 12/9 7.1) & $13 / 19$ & 0.372 \\
\hline Age (years) & $20.05 \pm 2.88$ & $21.29 \pm 3.33$ & $20.00 \pm 2.36$ & 0.154 \\
\hline $\mathrm{BMI}\left(\mathrm{kg} / \mathrm{m}^{2}\right)$ & $25.59 \pm 5.50$ & $28.57 \pm 5.30^{*}$ & $26.64 \pm 6.98$ & 0.039 \\
\hline Waist circumstance (cm) & $84.84 \pm 14.14$ & $92.19 \pm 13.39^{*}$ & $88.05 \pm 16.51$ & 0.038 \\
\hline FAT\% & $30.19 \pm 10.05$ & $35.33 \pm 12.71^{*}$ & $33.83 \pm 9.97^{\star}$ & 0.014 \\
\hline $\mathrm{SBP}(\mathrm{mm} \mathrm{Hg})$ & $114.05 \pm 13.35$ & $119.22 \pm 15.51$ & $119.82 \pm 16.09^{*}$ & 0.02 \\
\hline $\mathrm{DBP}(\mathrm{mm} \mathrm{Hg})$ & $72.62 \pm 10.21$ & $77.17 \pm 10.27^{\star}$ & $76.60 \pm 11.55^{\star}$ & 0.019 \\
\hline $\mathrm{TG}(\mathrm{mmol} / \mathrm{L})$ & $1.08 \pm 0.67$ & $1.90 \pm 2.42^{\star \star \star}$ & $1.32 \pm 0.62^{\#}$ & $<0.001$ \\
\hline LDL-C (mmol/L) & $2.50 \pm 0.74$ & $2.84 \pm 0.86^{\star}$ & $2.65 \pm 0.75$ & 0.074 \\
\hline $\begin{array}{l}\text { Total cholesterol } \\
(\mathrm{mmol} / \mathrm{L})\end{array}$ & $4.31 \pm 0.86$ & $4.62 \pm 1.36$ & $4.46 \pm 0.83$ & 0.207 \\
\hline $\mathrm{HDL}-\mathrm{C}(\mathrm{mmol} / \mathrm{L})$ & $1.43 \pm 0.32$ & $1.45 \pm 0.38$ & $1.44 \pm 0.29$ & 0.983 \\
\hline ALT (IU/L) & 19.05 (18.17 to 19.97$)$ & 22.03 (17.59 to 27.59$)$ & 19.86 (16.55 to 23.83 ) & 0.432 \\
\hline AST (IU/L) & 18.86 (18.35 to 19.40$)$ & 20.35 (17.81 to 23.24$)$ & 18.32 (16.45 to 20.41$)$ & 0.467 \\
\hline $\mathrm{Hs}-\mathrm{CRP}$ (mg/L) & 0.55 (0.47 to 0.65$)$ & 0.77 (0.37 to 1.59$)$ & 0.56 (0.31 to 1.03$)$ & 0.693 \\
\hline $\mathrm{HbA1c}(\%)$ & $5.32 \pm 0.29$ & $5.60 \pm 0.29^{\star \star \star}$ & $5.41 \pm 0.31^{\#}$ & $<0.001$ \\
\hline \multicolumn{5}{|l|}{ Glucose (mmol/L) } \\
\hline Fasting & $4.81 \pm 0.34$ & $5.74 \pm 0.15^{\star \star \star}$ & $5.01 \pm 0.30^{\star \star \# \# \#}$ & $<0.001$ \\
\hline 0.5 hour & $7.73 \pm 1.23$ & $9.14 \pm 1.43^{\star \star *}$ & $8.72 \pm 1.13^{\star \star \star}$ & $<0.001$ \\
\hline 2 hours & $5.71 \pm 0.98$ & $6.41 \pm 1.04^{\star \star}$ & $8.40 \pm 0.52^{\star \star \star \# \# \#}$ & $<0.001$ \\
\hline \multicolumn{5}{|l|}{ Insulin (mU/L) } \\
\hline Fasting & $6.72(6.30$ to 7.18$)$ & $9.83(7.20 \text { to } 13.41)^{\star}$ & $8.53(6.63$ to 10.98$)$ & 0.016 \\
\hline 0.5 hour & 71.49 (67.07 to 76.21$)$ & 65.58 (48.52 to 88.63$)$ & 68.90 (53.77 to 88.30$)$ & 0.832 \\
\hline 2 hours & 34.23 (31.95 to 36.67$)$ & 42.65 (30.72 to 59.20$)$ & $\begin{array}{l}94.28(72.28 \text { to } \\
122.98)^{\star \star \star \# \#}\end{array}$ & $<0.001$ \\
\hline \multicolumn{5}{|c|}{ Insulin resistance and beta-cell function $†$} \\
\hline HOMA-IR & 1.45 (1.38 to 1.53$)$ & $2.09(1.63 \text { to } 2.68)^{*}$ & 1.73 (1.42 to 2.12$)$ & 0.007 \\
\hline$|S|$ & 6.23 (5.93 to 6.53$)$ & 5.02 (4.03 to 6.27$)$ & $4.41(3.69 \text { to } 5.29)^{\star \star}$ & $<0.001$ \\
\hline HOMA- $\beta$ & $108.31(102.84$ to 114.04$)$ & $74.89(58.47 \text { to } 95.85)^{\star}$ & $104.06(85.30$ to 127.03$)$ & 0.016 \\
\hline IGI & 1.33 (1.24 to 1.42$)$ & 0.92 (0.66 to 1.27$)$ & $0.89(0.68 \text { to } 1.16)^{*}$ & 0.002 \\
\hline Dlo & 8.24 (7.75 to 8.76$)$ & $4.59(3.44 \text { to } 6.13)^{\star \star \star}$ & $3.93(3.10 \text { to } 4.98)^{\star \star \star}$ & $<0.001$ \\
\hline \multicolumn{5}{|l|}{ Adipokines } \\
\hline FGF21 (pg/ml) & 76.93 (69.49 to 85.17$)$ & $107.02(66.67$ to 171.78$)$ & $120.19(81.42 \text { to } 177.42)^{\star}$ & 0.045 \\
\hline RBP4 ( $\mu \mathrm{g} / \mathrm{mL})$ & 8.97 (8.68 to 9.27$)$ & 9.59 (8.20 to 11.22$)$ & 9.08 (8.00 to 10.31$)$ & 0.707 \\
\hline Leptin (ng/mL) & 1.78 (1.62 to 1.96$)$ & 2.48 (1.59 to 3.86$)$ & $2.67(1.86 \text { to } 3.82)^{*}$ & 0.043 \\
\hline Adiponectin ( $\mu \mathrm{g} / \mathrm{mL})$ & $6.78(6.41$ to 7.18$)$ & 6.88 (5.21 to 9.10$)$ & 5.41 (4.34 to 6.75$)$ & 0.148 \\
\hline Leptin/adiponectin & 26.32 (23.53 to 29.44$)$ & 35.57 (20.65 to 61.27$)$ & $49.33(32.09 \text { to } 75.82)^{\star \star}$ & 0.014 \\
\hline \multicolumn{5}{|l|}{ MetS components } \\
\hline Central obesity, n (\%) & $250(52.3)$ & $15(71.4)$ & $20(62.5)$ & 0.134 \\
\hline $\begin{array}{l}\mathrm{BP} \geq 130 / 85 \mathrm{~mm} \mathrm{Hg} \\
\mathrm{n}(\%)\end{array}$ & $80(16.7)$ & $8(38.1)^{\star}$ & $12(37.5)^{\star \star}$ & 0.001 \\
\hline $\mathrm{TG} \geq 1.7 \mathrm{mmol} / \mathrm{L}, \mathrm{n}(\%)$ & $62(13.0)$ & $4(19.0)$ & $9(28.1)^{*}$ & 0.045 \\
\hline Low HDL-C, n (\%) & $41(8.6)$ & $1(4.8)$ & $3(9.4)$ & 0.924 \\
\hline MetS, n (\%) & $36(7.5)$ & $3(14.3)$ & $15(46.9)^{\star \star \star \#}$ & $<0.001$ \\
\hline NAFLD, n (\%) & $127(28.5)$ & $12(57.1)^{\star \star}$ & $13(40.6)$ & 0.009 \\
\hline
\end{tabular}


Table 1 Continued

\begin{tabular}{lllll}
\hline & NGT & ilFG & ilGT & P value \\
\hline $\begin{array}{l}\text { NAFLD, moderate to } \\
\text { severe, } \mathrm{n}(\%)\end{array}$ & $40(9.0)$ & $3(14.3)$ & $9(28.1)^{\star *}$ & $\mathbf{0 . 0 0 3}$ \\
\hline
\end{tabular}

All values are reported as mean \pm SD or geometric mean $(95 \% \mathrm{Cl})$ unless otherwise indicated.

$P$ values were calculated from the analysis of covariance or general linear regression model with post hoc comparisons for continuous variables and $\chi^{2}$ test or Fisher exact test for categorical variables. Differences versus NGT are indicated as ${ }^{*} p<0.05$, ${ }^{* *} p<0.01$, and ${ }^{* * *} p<0.001$. Differences versus impaired fasting glucose are indicated as ${ }^{\#} p<0.05,{ }^{\# \#} p<0.01$, and ${ }^{\# \#} p<0.001$. Values in bold are significant at $\mathrm{p}<0.05$.

†Adjusted for age, gender and BMI.

ALT, alanine aminotransferase; AST, aspartate transaminase; BMI, body mass index; BP, blood pressure; DBP, diastolic blood pressure; Dlo, oral disposition index; FAT\%, fat mass percentage; FGF21, fibroblast growth factor 21; HbA1c, hemoglobin A1c; HDL-C, high-density lipoprotein cholesterol; HOMA- $\beta$, Homeostasis model assessment of $\beta$-cell function; HOMA-IR, homeostasis model assessment of insulin resistance; Hs-CRP, high-sensitivity $\mathrm{C}$ reactive protein; IGI, insulinogenic index; ilFG, isolated impaired fasting glucose; ilGT, isolated impaired glucose tolerance; ISI, insulin sensitivity index; LDL-C, low-density lipoprotein cholesterol; MetS, metabolic syndrome; NAFLD, nonalcoholic fatty liver disease; NGT, normal glucose tolerance; RBP4, retinol binding protein 4; SBP, systolic blood pressure; TG, triglyceride.

were $7.5 \%, 14.3 \%$, and $46.9 \%$ among the NGT, iIFG and iIGT groups, respectively. Compared with the NGT group, the iIFG group had a higher proportion of participants with hypertension $(\mathrm{p}<0.05)$, and the iIGT group had higher proportions with hypertension $(\mathrm{p}<0.01)$ and hypertriglyceridemia $(\mathrm{p}<0.05)$. Consequently, the iIGT group had more components of MetS than the iIFG group $(\mathrm{p}<0.001)$. Compared with the NGT group, the iIFG group had a higher prevalence of NALFD, while the iIGT group had the highest proportion with moderate-tosevere NALFD (all $\mathrm{p}<0.01$ ).

\section{Comparisons of IR and beta-cell function in participants with isolated IFG and IGT}

As shown in table 1, the homeostasis model indices (HOMA-IR and HOMA- $\beta$ ) were most aberrant in the iIFG group (all $\mathrm{p}<0.05$ ), while the iIGT group had significantly reduced ISI $(p<0.01)$ after adjustment for age, gender and BMI. The early-phase insulin secretion parameter, IGI, was significantly lower in the iIGT group compared with NGT $(\mathrm{p}<0.05)$, and after accounting for IR, betacell function (as reflected by DIo) gradually diminished across the three groups $(\mathrm{p}<0.001)$.

\section{Comparisons of adipokines in participants with ilFG and ilGT}

Among the four adipokines, including leptin, adiponectin, FGF21 and RBP4, as well as leptin:adiponectin ratio (table 1), only the iIGT group showed significant differences in the levels of FGF21, leptin and leptin/adiponectin compared with the NGT group. After adjusting for age and gender (figure 1), adiponectin levels were significant lower in the iIGT versus NGT and iIFG groups (all $\mathrm{p}<0.05$ ), whereas the difference in leptin levels between the iIGT and NGT groups was ablated $(p>0.05)$. When further controlling for BMI, adiponectin levels remained significantly lower in the iIGT than the NGT $(\mathrm{p}<0.05)$ and iIFG $(\mathrm{p}=0.01)$ groups, while the differences in FGF21 levels between the NGT and iIGT groups were attenuated $(\mathrm{p}=0.064)$.
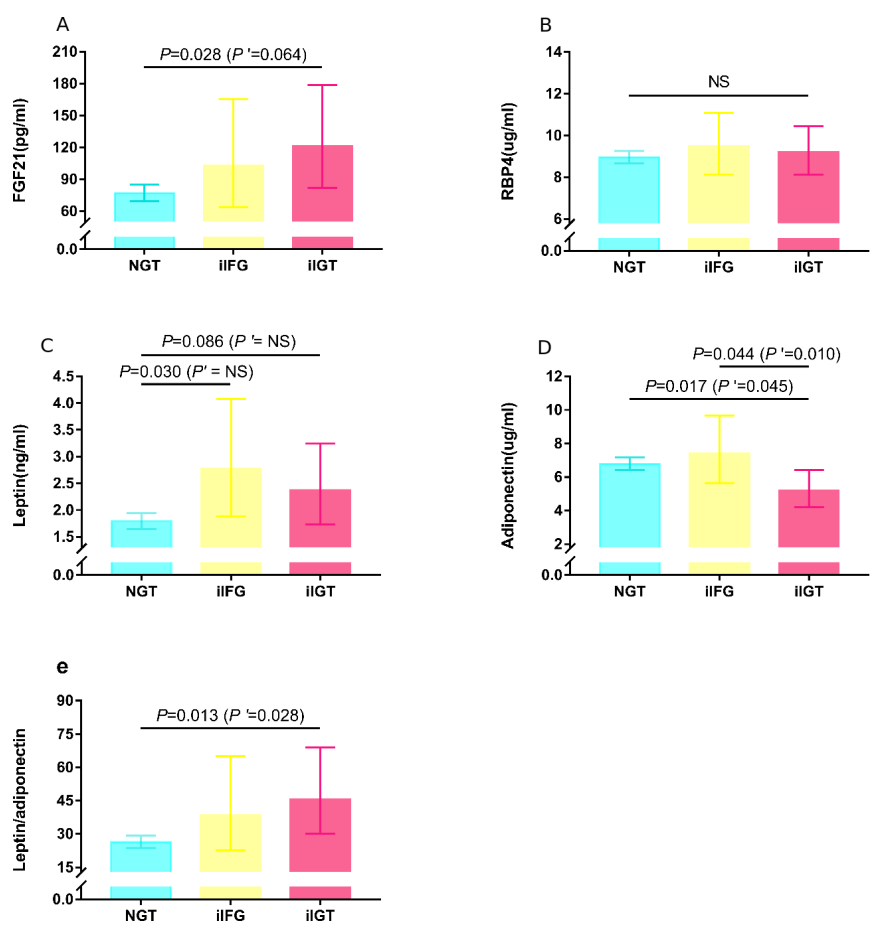

Figure 1 Figure 1Comparison of adipokine levels among the three groups. Data were presented as geometric mean $(95 \% \mathrm{Cl})$ and calculated from the general linear regression model with post hoc comparisons after controlling for age and gender. P' values were further adjusted for BMI. Only $p$ values of $<0.10$ were represented. (A) The ilGT group had higher FGF21 levels than the NGT group, and the difference was attenuated with further adjustment for BMI. (B) RBP4 levels were not significantly different (NS) among the three groups. (C) The difference in leptin levels among the three groups was ablated after controlling for BMI. (D) The ilFG group was associated with decreased adiponectin levels compared with NGT and ilFG. (E) The ilGT group was associated with increased leptin/adiponectin compared with the NGT group. FGF21, fibroblast growth factor 21; ilFG, isolated impaired fasting glucose; ilGT, isolated impaired glucose tolerance; NGT, normal glucose tolerance; NS, not significant; RBP4, retinol binding protein 4. 
Table 2 ORs of cardiometabolic risk factors comparing participants with ilFG or ilGT versus NGT

\begin{tabular}{|c|c|c|c|c|c|}
\hline & & iIFG & & iIGT & \\
\hline & NGT & OR $(95 \% \mathrm{Cl})$ & $P$ value & OR $(95 \% \mathrm{Cl})$ & $P$ value \\
\hline Central obesity & 1 (ref.) & 1.08 (0.23 to 5.13$)$ & 0.924 & 2.44 (0.71 to 8.35$)$ & 0.156 \\
\hline $\mathrm{BP} \geq 130 / 85 \mathrm{~mm} \mathrm{Hg}$ & 1 & 2.41 (0.86 to 6.76$)$ & 0.095 & 3.99 (1.46 to 10.93$)$ & 0.007 \\
\hline $\mathrm{TG} \geq 1.7 \mathrm{mmol} / \mathrm{L}$ & 1 & 0.91 (0.28 to 3.00$)$ & 0.876 & 2.40 (0.95 to 6.07$)$ & 0.065 \\
\hline $\mathrm{HDL}-\mathrm{C}<1.04 \mathrm{mmol} / \mathrm{L}$ & 1 & 0.36 (0.05 to 2.97$)$ & 0.345 & 0.78 (0.20 to 3.05$)$ & 0.721 \\
\hline HOMA-IR >3.0 & 1 & 3.50 (1.19 to 10.30$)$ & 0.023 & 3.65 (1.37 to 9.73 ) & 0.010 \\
\hline Metabolic syndrome & 1 & 1.36 (0.33 to 5.64$)$ & 0.674 & 32.60 (9.66 to 110.02$)$ & $<0.001$ \\
\hline NAFLD & & & & & \\
\hline Mild & 1 & 2.17 (0.65 to 7.26$)$ & 0.208 & $1.10(0.24$ to 4.95$)$ & 0.902 \\
\hline Moderate to severe & 1 & 5.31 (0.71 to 39.41$)$ & 0.103 & 6.29 (1.04 to 37.86$)$ & 0.045 \\
\hline
\end{tabular}

Logistic regression models were used with adjustment for age, gender and body mass index.

$P$ values in bold are significant at $p<0.05$.

BP, blood pressure; HDL-C, high-density lipoprotein cholesterol; HOMA-IR, homeostasis model assessment of insulin resistance; ilFG, isolated impaired fasting glucose; ilGT, isolated impaired glucose tolerance; NAFLD, non-alcoholic fatty liver disease; NGT, normal glucose tolerance; ref., reference; TG, triglyceride.

\section{Comparisons of cardiometabolic risk factors in participants with illFG versus ilGT}

Compared with the NGT group, the iIFG group had higher risk of exhibiting hypertension (BP $\geq 130 / 85 \mathrm{~mm}$ $\mathrm{Hg}$ ), IR (defined as HOMA-IR $>3.0$ ) and mild NAFLD, while the iIGT group displayed a higher risk of hypertension, hypertriglyceridemia $(\geq 1.7 \mathrm{mmol} / \mathrm{L})$, IR, MetS and moderate-to-severe NAFLD after controlling for age and gender (all $\mathrm{p}<0.05$, data not shown). However, after further adjusting for BMI (table 2), only the elevated risk of IR (OR=3.50, 95\% CI 1.19 to $10.30, p=0.023$ ) remained for the iIFG group, while the increased risks of hypertension, IR, MetS and the moderate-to-severe NAFLD remained significant in the iIGT group, with odds ratios of 4.0 (95\% CI 1.46 to $10.93, \mathrm{p}=0.007$ ), 3.7 (95\% CI 1.37 to $9.73, \mathrm{p}=0.01), 32.6$ (95\% CI 9.67 to $110.02, \mathrm{p}<0.001$ ), and 6.29 (95\% CI 1.04 to 37.86 , $\mathrm{p}=0.045)$, respectively.

\section{Characterizing the cardiometabolic risk factors in} participants with normal FPG but with missed IGT by FPG test to inform a strategy for screening at-risk youth for prediabetes

Given our evidence that FPG is inadequate for the detection of IGT, we endeavored to assess the cardiometabolic risk profile in the subgroup of participants who had normal FPG but were still likely to have IGT. Table 3 demonstrates that in the 510 participants with normal FPG, the odds of an abnormal $2 \mathrm{~h}$ PG increased 2.9-fold in participants with hypertriglyceridemia, 4.7-fold with hypertension, 4.7-fold with moderate-to-severe NAFLD and 18.3-fold in the presence of MetS. However, IGT

Table 3 ORs of IGT according to metabolic risk factors in those with normal fasting plasma glucose $(\mathrm{N}=510)$

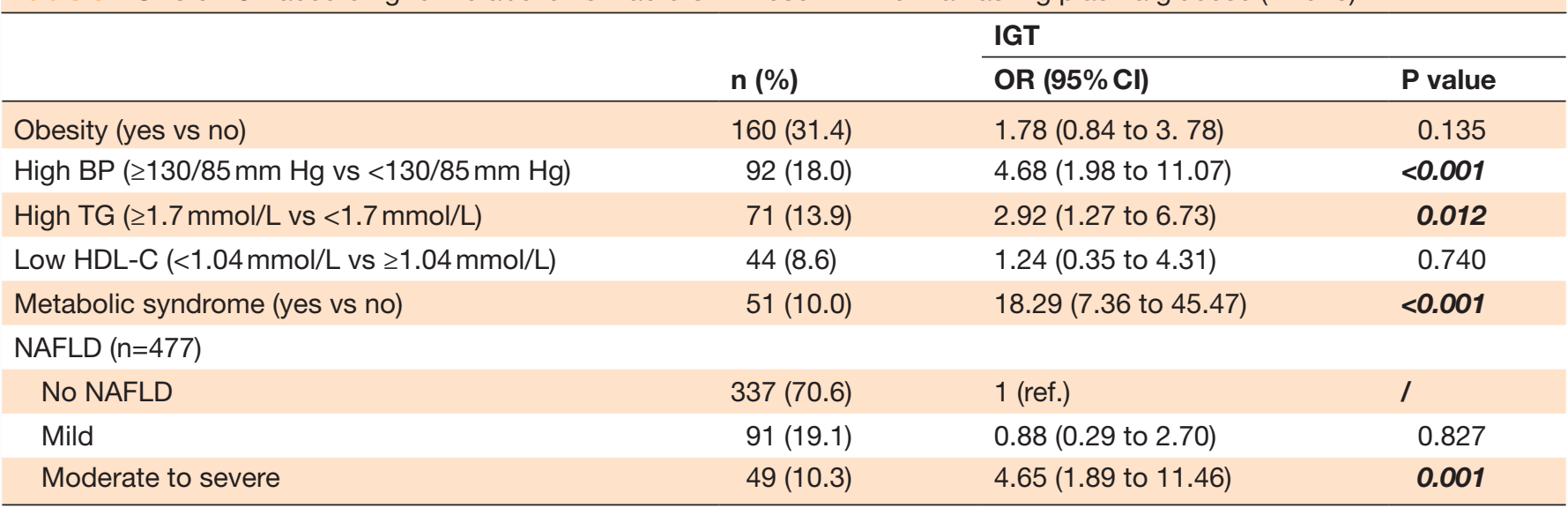

Logistic regression models were used with adjustment for age and gender.

$P$ values in bold are significant at $p<0.05$.

BP, blood pressure; HDL-C, high-density lipoprotein cholesterol; IGT, impaired glucose tolerance; NAFLD, non-alcoholic fatty liver disease; ref., reference; TG, triglyceride. 


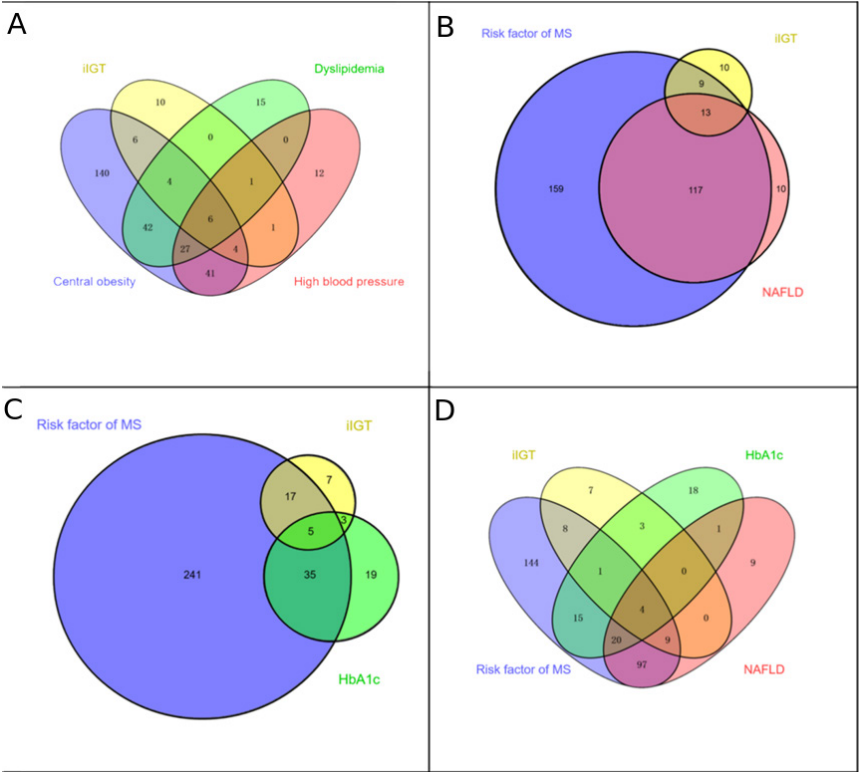

Figure 2 Comparisons of combination efficiency to identify the missed IGT in those with normal FPG. Proportions and their overlap between screening with risk of MetS (including any classical risk factor, i.e, central obesity, dyslipidaemia and high blood pressure), NAFLD, HbA1c of $\geq 5.7 \%$ and ilGT (FPG of $<5.6 \mathrm{mmol} / \mathrm{L}$ and 2-hour PG of $\geq 7.8-11.1 \mathrm{mmol} / \mathrm{L}$ ). Screening subjects with any components of MetS $(A)$ and NAFLD (B) would miss $31 \%$ (10/32) of participants with IGT; further adding $\mathrm{HbA} 1 \mathrm{c}(\geq 5.7 \%)$, in addition to the risk factor of MetS (C) and NAFLD screening (D), 22\% (7/32) of participants with IGT remained to be missed. FPG, fasting plasma glucose; HbA1c, haemoglobin A1c; IGT, impaired glucose tolerance; ilGT, isolated impaired glucose tolerance; MetS, metabolic syndrome; NAFLD, non-alcoholic fatty liver disease.

was not significantly more prevalent among subjects with obesity. Therefore, our findings suggest that among youth with normal FPG, the presence of hypertension, hypertriglyceridemia, moderate-to-severe NAFLD or MetS increases the likelihood of IGT, such that a 2-hour OGTT is warranted.

However, it should be noted that even with this approach, 31\% (10/32) of participants with IGT would be missed (figure 2A,B). Moreover, the addition of HbA1c $(\geq 5.7 \%)$ and the components of MetS (figure 2C) or NAFLD to the screening paradigm (figure 2D) only garners an additional three subjects with IGT, leaving $22 \%(7 / 32)$ of participants to be missed.

\section{DISCUSSION}

In this cross-sectional study of adolescents and young adults (mean age of 20 years) at risk of MetS, we demonstrate surprisingly poor agreement between FPG and OGTT in the classification of pre-diabetes and diabetes when compared with previous reports in older adults. FPG failed to detect nearly all cases of IGT, despite the fact that IGT was indicative of greater IR and beta-cell dysfunction, as well as a more adverse cardiometabolic risk factor profile, than was IFG. Although FPG is the most common screening procedure for dysglycemia in clinical practice, for Chinese youths with existing indicators of MetS and normal FPG, a 2-hour OGTT is necessary to identify the pre-diabetes phenotype. To the best of our knowledge, this is the first study to compare the relative value of FPG versus 2-hour OGTT to identify subphenotypes of pre-diabetes in Chinese youth and to explore the pathophysiological mechanisms responsible for their elevated cardiometabolic risk.

Although experts do not agree on how to identify individuals at high risk of diabetes, since the ADA advocated for the addition of HbAlc to FPG and $2 \mathrm{~h} \mathrm{PG}$ for the screening and diagnosis of pre-diabetes in $2010,{ }^{37}$ there has been increased interest in establishing thresholds for HbA1c, FPG and 2-hour OGTT in different ethnic population. $^{339}$ A study of 6884 adults from Thailand showed that FPG failed to identify $46.3 \%$ of all pre-diabetes and $54.7 \%$ of diabetes cases, whereas the corresponding percentages for missed diagnosed using OGTT were just $18.9 \%$ and $7.0 \%$, respectively. ${ }^{40}$ In 2012, Sesti et al ${ }^{41}$ demonstrated discordance between HbAlc, FPG, and 2-hour OGTT for the identification of pre-diabetes and that cardiometabolic risk profiles vary depending on the defining metabolic parameter, with 2-hour OGTT showing the stronger association with cardiometabolic risk factors and subclinical atherosclerosis than FPG or HbAlc. Considering the discrepancies in screening recommendations for dysglycemia, ${ }^{3} 1699$ we examined the relative merit of FPG versus 2-hour OGTT when screening for pre-diabetes and T2D in a young Chinese population. We found a greater proportion of hyperglycemia was missed by FPG compared with 2-hour OGTT, and the presence of hypertension, hypertriglyceridemia, NAFLD or MetS increases the risk of IGT in participants, even when FPG is normal. So, consistent with a study of 563 obese Italian children and adolescents where IFG was not a good predictor of IGT in youth, ${ }^{42}$ in the presence of indicators of MetS, FPG is inadequate as a screen for the pre-diabetes phenotype.

IR and beta-cell dysfunction are fundamental pathophysiological mechanisms for diabetes, which also play crucial roles in determining pre-diabetes. ${ }^{6}$ A growing number of studies examining the pathogenesis of prediabetes, primarily in older adults, ${ }^{5-9}$ suggest that IFG is characterized by a combination of hepatic IR and defective early-phase insulin secretion, whereas IGT is defined by IR in skeletal muscle rather than liver, combined with defective early and late-phase insulin secretion. In contrast, few studies have examined the mechanisms responsible for pre-diabetes in youth, despite the likelihood that the pathophysiology may be more readily elucidated in the absence of confounding comorbid factors. In the present study, we found that those with IFG had an elevated HOMA-IR, while the IGT group had reduced ISI compared with their NGT counterparts. Considering that HOMA-IR index mainly reflects hepatic IR and ISI reflects both hepatic and peripheral 
(ie, muscle) insulin sensitivity, our findings in youth are consistent with previous reports in adults. ${ }^{5-9}$ In addition, impaired beta-cell function was evident from a decreased DIo in those with IFG and IGT versus NGT, suggesting an early and significant decline in beta-cell function, which was observed in a previous study in obese adolescents. ${ }^{43}$

Individuals with pre-diabetes are at high risk, not only for the development of diabetes mellitus ${ }^{44}$ but also for adverse cardiovascular outcomes. ${ }^{10} 45$ Moreover, pre-diabetes subphenotypes (ie, IFG vs IGT) may be associated with disparate risks of microvascular and macrovascular complications. For example, the Baltimore Longitudinal Study on Ageing demonstrated that IGT, but not IFG, was associated with increased levels of coronary heart disease risk factors. ${ }^{46}$ Similar findings were recently reported in a large European cohort with coronary artery disease ${ }^{47}$ and a recent meta-analysis of 53 prospective cohort studies. ${ }^{45}$ While these associations have not been well studied in youth, our data suggest that IGT portends a higher likelihood of hypertension, hypertriglyceridemia, NAFLD and MetS in young Chinese individuals than does IFG. Consistent with our result, a recent study in 3088 Italian children and adolescents with overweight/obesity demonstrated that IGT was associated with higher cardiometabolic risk than IFG. ${ }^{48}$ Thus, it appears that pathophysiological mechanisms leading to cardiovascular disease among pre-diabetics are not strongly influenced by age or ethnicity.

The increased risk of cardiometabolic disease in prediabetes is multifactorial. ${ }^{49}$ Certainly, the higher degree of IR exhibited by IGT individuals may contribute to the development of atherosclerosis. In addition, chronic subclinical inflammation, evidenced by adipokine imbalance, could be a unifying mechanistic factor because it predicts the development of both diabetes and cardiovascular disease. ${ }^{12}{ }^{14}$ We examined this hypothesis by exploring the association between the pre-diabetes subphenotypes and four well-known IR-related adipokines (leptin, adiponectin, FGF21, and RBP4). ${ }^{14}$ Even after controlling for BMI, higher levels of FGF21 and leptin/adiponectin ratio, as well as lower adiponectin were present in the IGT (but not the IFG) group compared with their NGT counterparts, suggesting a specific role of adipose tissue dysfunction in the pathogenesis of IGT. Our findings are congruent with a number of studies ${ }^{50-52}$ reporting various adipokine abnormalities associated with IGT compared with NGT. Although minor discrepancies exist between which adipokines are affected (and to what degree), this can be explained by differences in age, sample size and ethnicity of participants. Our findings suggest that alterations in blood levels of certain adipokines are already detectable in youth with pre-diabetes, particularly those with IGT status, and may reflect adipose tissue dysfunction as an early pathogenic event in T2D development. Thus, circulating adipokines may be a more sensitive biomarker of adipose tissue dysfunction than anthropometric measures like BMI or WC. However, clearly more studies in younger populations are needed, since data in this age group are limited.

Given our evidence that IGT is representative of more serious IR, beta-cell dysfunction, and greater cardiometabolic risk than is IFG, and isolated use of FPG is limited by its discordance with IGT, we sought a method to efficiently identify the obscured IGT in those initially classified as NGT on the basis of FPG alone. In line with the new guidelines of ADA-2019, ${ }^{17}$ we found that IGT occurs more frequently in youth with conditions associated with IR (including hypertension and dyslipidemia) and in those with NAFLD based on liver ultrasound. When 2-hour OGTT was performed in youth meeting criteria for the MetS in their initial screening, more than two-thirds of IGT can be detected. This two-stage screening process is less labor intensive and might translate into more efficient clinical practice by avoiding the OGTT in $59 \%$ of participants with NGT. However, it should be noted that even with this strategy, nearly a third of individuals with IGT would be missed. Adding HbAlc testing in individuals with traditional risk factors of the MetS leaves only $22 \%$ of undetected IGT to be missed. It is open to debate whether this is acceptable, in light of the possibility of avoiding unnecessary OGTTs in low-risk individuals. Previous studies of western children and adolescents have proposed that using fasting TG levels alone ${ }^{53}$ or in combination with $\mathrm{FPG}^{54}$ identifies the subsample in whom to perform OGTT. However, these approaches were less than satisfactory in our cohort of youth (see online supplementary file 1). Moreover, compared with TG, BP appears be of greater value when predicting IGT in our cohort (table 3 and figure 1). This dichotomy is likely to be related to differences in age and ethnicity in their respective cohorts, but further study is needed to define optimal screening strategies.

Strengths of this study include its relatively large cohort, standardized collection of detailed data to assess cardiometabolic phenotype, and comprehensive approach to screen for dysglycemia via OGTT. Despite these strengths, some limitations remain. First, the estimation of IR and beta-cell function using OGTT is less precise than 'gold standard' measures, like euglycemic clamp or frequently sampled intravenous glucose tolerance test. However, ISI, HOMA-IR, and DIo have been validated in other populations against these standards and found to be valid and appropriate for clinical investigations and epidemiological studies. ${ }^{31} 3255$ It should be noted, however, that we only used three time points for glucose and insulin during the OGTT to calculate the ISI $_{\text {Matsuda }}$, which may lead to inaccuracy compared with the original calculation, which is based on the mean of five values. ${ }^{31}$ Second, as our sample was taken from a pool of Chinese youths at increased risk of cardiovascular disease, similar studies in other ethnical populations are needed to evaluate the generalizability of our findings. Finally, while we found that youth with 
IGT possessed markers for increased cardiometabolic risk, a causal relationship is not possible to infer due to the cross-sectional nature of the study design. However, our ongoing follow-up observations in this cohort may serve to elucidate a causal mechanism.

\section{CONCLUSIONS}

In conclusion, in this young Chinese population, we found that FPG fails to identify nearly all individuals with IGT. The fact that IGT was more indicative than IFG of profound IR, $\beta$-cell dysfunction and an adverse cardiometabolic profile suggests that FPG is inadequate as a screen for dysglycemia in Chinese youth. Rather, a 2-hour OGTT is necessary to adequately identify prediabetes in the subset of subjects with risk factors for MetS, even in the absence of obesity.

\section{Author affiliations}

'Department of Endocrinology, NHC Key Laboratory of Endocrinolog, Peking Union Medical College Hospital,Chinese Academy of Medical Sciences \& Peking Union Medical College, Beijing, China

${ }^{2}$ Department of Endocrinology, Beijing Chaoyang Hospital,Capital Medical University, Beijing, China

${ }^{3}$ Health Weight Program, The Children's Hospital of Philadelphia, Perelman School of Medicine at University of Pennsylvania, Philadelphia, Pennsylvania, USA

${ }^{4}$ Epidemiology Department, Fielding School of Public Health, University of California Los Angeles, Los Angeles, California, USA

${ }^{5}$ Division of Endocrinology, The Children's Hospital of Philadelphia, Perelman School of Medicine, University of Pennsylvania, Philadelphia, Pennsylvania, USA

Acknowledgements The authors thank Professor Jie Mi and all the Beijing Child and Adolescent Metabolic Syndrome study members, and all participants for their continuing participation in this research effort.

Contributors YL and DF contributed to the data analysis and drafted the manuscript; ICE and YZ contributed to the data analysis and revised the manuscript; LH, YZ, JF, GL, DW and YW contributed to the data collection; SMW contributed to the concept and design of the study, interpretation of the data and revision of the manuscript; ML contributed to the concept, design of the study, analysis of the data and revision of the manuscript. SG was responsible for the concept, design and data collection in the Beijing Child and Adolescent Metabolic Syndrome follow-up study; contributed to the acquisition and interpretation of the data; and revised the manuscript. All authors read and approved the final manuscript.

Funding The authors have not declared a specific grant for this research from any funding agency in the public, commercial or not-for-profit sectors.

Competing interests None declared.

Patient consent for publication Not required.

Ethics approval The study protocol was approved by the Ethics Committee at the Beijing Chaoyang Hospital, and signed informed consent was obtained from all participants and/or their parents or guardians.

Provenance and peer review Not commissioned; internally peer reviewed.

Data availability statement All data relevant to the study are included in the article or uploaded as supplementary information.

Open access This is an open access article distributed in accordance with the Creative Commons Attribution Non Commercial (CC BY-NC 4.0) license, which permits others to distribute, remix, adapt, build upon this work non-commercially, and license their derivative works on different terms, provided the original work is properly cited, appropriate credit is given, any changes made indicated, and the use is non-commercial. See: http://creativecommons.org/licenses/by-nc/4.0/.

\section{ORCID iDs}

Ming Li http://orcid.org/0000-0002-8427-605X

Steven M Willi http://orcid.org/0000-0002-4910-2425
REFERENCES

1 International Diabetes Federation. IDF DIABETES ATLAS - 8TH EDITION. Available: http://www.diabetesatlas.org [Accessed 23 May 2018].

$2 \mathrm{Xu} \mathrm{Y,} \mathrm{Wang} \mathrm{L,} \mathrm{He} \mathrm{J,} \mathrm{et} \mathrm{al.} \mathrm{Prevalence} \mathrm{and} \mathrm{control} \mathrm{of} \mathrm{diabetes} \mathrm{in}$ Chinese adults. JAMA 2013;310:948-59.

3 Barry E, Roberts S, Oke J, et al. Efficacy and effectiveness of screen and treat policies in prevention of type 2 diabetes: systematic review and meta-analysis of screening tests and interventions. BMJ 2017;356:i6538.

4 American Diabetes Association. Standards of medical care in diabetes-2014. Diabetes Care 2014;37:S14-80.

5 Abdul-Ghani MA, Jenkinson CP, Richardson DK, et al. Insulin secretion and action in subjects with impaired fasting glucose and impaired glucose tolerance: results from the Veterans administration genetic epidemiology study. Diabetes 2006;55:1430-5.

6 Abdul-Ghani MA, Tripathy D, DeFronzo RA. Contributions of beta-cell dysfunction and insulin resistance to the pathogenesis of impaired glucose tolerance and impaired fasting glucose. Diabetes Care 2006;29:1130-9.

7 Abdul-Ghani M, DeFronzo RA, Jayyousi A. Prediabetes and risk of diabetes and associated complications: impaired fasting glucose versus impaired glucose tolerance: does it matter? Curr Opin Clin Nutr Metab Care 2016;19:394-9.

8 Kanat M, Mari A, Norton L, et al. Distinct $\beta$-cell defects in impaired fasting glucose and impaired glucose tolerance. Diabetes 2012;61:447-53.

9 Ferrannini E, Gastaldelli A, Miyazaki Y, et al. Beta-Cell function in subjects spanning the range from normal glucose tolerance to overt diabetes: a new analysis. J Clin Endocrinol Metab 2005;90:493-500.

10 DeFronzo RA, Abdul-Ghani M. Assessment and treatment of cardiovascular risk in prediabetes: impaired glucose tolerance and impaired fasting glucose. Am J Cardiol 2011;108:3B-24.

11 Evans JMM, Eades CE, Leese GP. The risk of total mortality and cardiovascular mortality associated with impaired glucose regulation in Tayside, Scotland, UK: a record-linkage study in 214094 people. BMJ Open Diabetes Res Care 2015;3:e000102.

12 Bensinger SJ, Tontonoz P. Integration of metabolism and inflammation by lipid-activated nuclear receptors. Nature 2008;454:470-7.

13 Weiss R, Santoro N, Giannini C, et al. Prediabetes: the importance of early identification and intervention. The Lancet 2017;1:240-8.

14 Mechanick JI, Zhao S, Garvey WT. The Adipokine-CardiovascularLifestyle network: translation to clinical practice. J Am Coll Cardiol 2016;68:1785-803.

$15 \mathrm{Li} \mathrm{S}$, Shin HJ, Ding EL, et al. Adiponectin levels and risk of type 2 diabetes: a systematic review and meta-analysis. JAMA 2009;302:179-88.

16 Cameron FJ, Wherrett DK. Care of diabetes in children and adolescents: controversies, changes, and consensus. Lancet 2015;385:2096-106.

17 American Diabetes Association. Classification and diagnosis of diabetes: standards of medical care in Diabetes-2019. Diabetes Care 2019;42:S13-28.

18 Fu J, Hou C, Li L, et al. Vitamin D modifies the associations between circulating betatrophin and cardiometabolic risk factors among Youths at risk for metabolic syndrome. Cardiovasc Diabetol 2016;15:142.

19 Feng D, Zhang J, Fu J, et al. Association between sleep duration and cardiac structure in youths at risk for metabolic syndrome. Sci Rep 2016;6:39017.

20 Li M, Fisette A, Zhao X-Y, et al. Serum resistin correlates with central obesity but weakly with insulin resistance in Chinese children and adolescents. Int J Obes 2009;33:424-39.

21 Li L, Yin J, Cheng H, et al. Identification of genetic and environmental factors predicting metabolically healthy obesity in children: data from the BCAMS study. J Clin Endocrinol Metab 2016;101:1816-25.

22 Jian-gao F. Guidelines for management of nonalcoholic fatty liver disease: an updated and revised edition. Zhonghua Gan Zang Bing Za Zhi 2010;18:163-6.

23 Mehmood S, Margolis M, Ye C, et al. Hepatic fat and glucose tolerance in women with recent gestational diabetes. BMJ Open Diabetes Res Care 2018:6:e000549.

24 Saadeh S, Younossi ZM, Remer EM, et al. The utility of radiological imaging in nonalcoholic fatty liver disease. Gastroenterology 2002;123:745-50.

25 Li M, Wu C, Song A, et al. Development and preliminary application of enzyme-linked immunosorbent assay for human net insulin in serum. Chin J Endocrinol Metab 1997;5:214-7. 
26 Li M, Yin J-hua, Zhang K, et al. [A highly sensitive enzyme-linked immunosorbent assay for measurement of leptin secretion in human adipocytes]. Zhonghua Yi Xue Za Zhi 2008;88:3293-7.

$27 \mathrm{Li}$ Q, Lu Y, Sun L, et al. Plasma adiponectin levels in relation to prognosis in patients with angiographic coronary artery disease. Metabolism 2012;61:1803-8.

28 Li G, Esangbedo IC, Xu L, et al. Childhood retinol-binding protein 4 (RBP4) levels predicting the 10-year risk of insulin resistance and metabolic syndrome: the BCAMS study. Cardiovasc Diabetol 2018;17:69.

29 Li G, Feng D, Qu X, et al. Role of adipokines FGF21, leptin and adiponectin in self-concept of youths with obesity. Eur Neuropsychopharmacol 2018;28:892-902.

30 Matthews DR, Hosker JP, Rudenski AS, et al. Homeostasis model assessment: insulin resistance and beta-cell function from fasting plasma glucose and insulin concentrations in man. Diabetologia 1985;28:412-9.

31 Matsuda M, DeFronzo RA. Insulin sensitivity indices obtained fro M oral glucose tolerance testing. Diabetes care 1999;22:1462-70.

32 George L, Bacha F, Lee S, et al. Surrogate estimates of insulin sensitivity in obese youth along the spectrum of glucose tolerance from normal to prediabetes to diabetes. $J$ Clin Endocrinol Metab 2011;:96:2136-45

33 Yeckel CW, Weiss R, Dziura J, et al. Validation of insulin sensitivity indices from oral glucose tolerance test parameters in obese children and adolescents. J Clin Endocrinol Metab 2004;89:1096-101.

34 Gungor N, Saad R, Janosky J, et al. Validation of surrogate estimates of insulin sensitivity and insulin secretion in children and adolescents. J Pediatr 2004;144:47-55.

35 Phillips DI, Clark PM, Hales CN, et al. Understanding oral glucose tolerance: comparison of glucose or insulin measurements during the oral glucose tolerance test with specific measurements of insulin resistance and insulin secretion. Diabet Med 1994;11:286-92.

36 Kahn SE, Prigeon RL, McCulloch DK, et al. Quantification of the relationship between insulin sensitivity and beta-cell function in human subjects. Evidence for a hyperbolic function. Diabetes 1993;42:1663-72.

37 American Diabetes Association. Standards of medical care in diabetes--2013. Diabetes Care 2013;36:S11-66.

38 Alberti KGMM, Eckel RH, Grundy SM, et al. Harmonizing the metabolic syndrome: a joint interim statement of the International diabetes Federation Task force on epidemiology and prevention; National heart, lung, and blood Institute; American heart association; world heart Federation; international atherosclerosis Society; and international association for the study of obesity. Circulation 2009;120:1640-5

39 Echouffo-Tcheugui JB, Ali MK, Griffin SJ, et al. Screening for type 2 diabetes and dysglycemia. Epidemiol Rev 2011;33:63-87.

40 Aekplakorn W, Tantayotai V, Numsangkul S, et al. Detecting prediabetes and diabetes: agreement between fasting plasma glucose and oral glucose tolerance test in Thai adults. J Diabetes Res 2015;2015:1-7.

41 Marini MA, Succurro E, Castaldo E, et al. Cardiometabolic risk profiles and carotid atherosclerosis in individuals with prediabetes identified by fasting glucose, postchallenge glucose, and hemoglobin A1c criteria. Diabetes Care 2012;35:1144-9.

42 Maffeis C, Pinelli L, Brambilla P, et al. Fasting plasma glucose (Fpg) and the risk of impaired glucose tolerance in obese children and adolescents. Obesity 2010;18:1437-42.

43 Cali' AMG, Bonadonna RC, Trombetta M, et al. Metabolic abnormalities underlying the different prediabetic phenotypes in obese adolescents. J Clin Endocrinol Metab 2008;93:1767-73.

44 de Vegt F, Dekker JM, Jager A, et al. Relation of impaired fasting and postload glucose with incident type 2 diabetes in a Dutch population: the Hoorn study. JAMA 2001;285:2109-13.

45 Huang Y, Cai X, Mai W, et al. Association between prediabetes and risk of cardiovascular disease and all cause mortality: systematic review and meta-analysis. BMJ 2016;355:i5953.

46 Blake DR, Meigs JB, Muller DC, et al. Impaired glucose tolerance, but not impaired fasting glucose, is associated with increased levels of coronary heart disease risk factors. DIABETES 2004;53:2005-100.

47 Shahim B, De Bacquer D, De Backer G, et al. The Prognostic Value of Fasting Plasma Glucose, Two-Hour Postload Glucose, and $\mathrm{HbA}_{1}$ in Patients With Coronary Artery Disease: A Report From EUROASPIRE IV: A Survey From the European Society of Cardiology. Diabetes Care 2017;40:1233-40.

48 Di Bonito P, Pacifico L, Chiesa C, et al. Impaired fasting glucose and impaired glucose tolerance in children and adolescents with overweight/obesity. J Endocrinol Invest 2017;40:409-16.

49 Hsueh WA, Orloski L, Wyne K. Prediabetes: the importance of early identification and intervention. Postgrad Med 2010;122:129-43.

$50 \mathrm{Li} \mathrm{H}, \mathrm{Bao} \mathrm{Y}, \mathrm{Xu} \mathrm{A}$, et al. Serum fibroblast growth factor 21 is associated with adverse lipid profiles and gammaglutamyltransferase but not insulin sensitivity in Chinese subjects. $J$ Clin Endocrinol Metab 2009;94:2151-6.

51 Nguyen PAH, Heggermont WA, Vanhaverbeke M, et al. Leptinadiponectin ratio in pre-diabetic patients undergoing percutaneous coronary intervention. Acta Cardiol 2015;70:640-6.

52 Tönjes A, Fasshauer M, Kratzsch J, et al. Adipokine pattern in subjects with impaired fasting glucose and impaired glucose tolerance in comparison to normal glucose tolerance and diabetes. PLoS One 2010;5:e13911.

53 Morrison KM, Xu L, Tarnopolsky M, et al. Screening for dysglycemia in overweight youth presenting for weight management. Diabetes Care 2012;35:711-6.

54 Morandi A, Maschio M, Marigliano M, et al. Screening for impaired glucose tolerance in obese children and adolescents: a validation and implementation study. Pediatr Obes 2014;9:17-25.

55 Burns SF, Bacha F, Lee SJ, et al. Declining $\beta$-cell function relative to insulin sensitivity with escalating OGTT 2-h glucose concentrations in the nondiabetic through the diabetic range in overweight youth. Diabetes Care 2011;34:2033-40. 